\title{
In vivo demonstration of cytosolic calcium level shift by oxalates and cancer
}

\author{
Abraham A. Embi ${ }^{1}$, Benjamin J. Scherlag ${ }^{2}$, Manuel Menes ${ }^{3}$, Sunny S. Po ${ }^{2}$ \\ 1. Independent, Miami Florida, USA. 2. Health Sciences Center, Heart Rhythm Research Institute, Department of \\ Medicine, University of Oklahoma, Oklahoma City, Oklahoma, USA. 3. Pathopinion Laboratory, Delray Beach, Florida, USA \\ Correspondence: Abraham A. Embi. Address: 13442 SW 102 LANE, Miami, FL 33186, USA. E-mail: Abe@embi.net
}

Received: December 20, 2012

Accepted: January 27, 2013

Online Published: January 31, 2013

DOI : $10.5430 /$ jst.v3n2p13

URL: http://dx.doi.org/10.5430/jst.v3n2p13

\begin{abstract}
A series of experiments on the effects of oxalates on the dog's heart sympathetic system resulted in the infiltration of calcium chelators into the adjacent atrial myocardium. The chelation process converts the ionic calcium into calcium carbonate $\left(\mathrm{CaCO}_{3}\right)$. We are presenting in vivo obtained images of the atrial myocyte cytoplasmic chelated calcium structures or mineralization. An increase of intracellular ionic calcium $\mathrm{Ca}^{++}$is known to trigger myocytes' hypertrophy in transgenic mice. We are introducing a new in vivo approach that also changes intracellular ionic calcium stores. This change of the available cytoplasmic ionic calcium stores was accomplished via oxalate induced calcium chelation or sequestration. We demonstrate that the process of chelation causes an increase in the cytosolic $\mathrm{Ca}^{++}$level (a stress on the endoplasmic reticulum) which could affect the normal myocardial ionic cellular syncytial flow through gap junctions. Representative biopsied stained images via the von Kossa calcium specific technique are presented. The mineralized calcium stores are also seen physiologically coalescing against the intercalated disc. We discuss the role that the molecular size of the calcium salt plays on the interruption of normal syncytial flow through the gap junction, and the consequences of chelation on cell death.
\end{abstract}

\section{Key words}

Syncytial flow; Calcium chelation; Cell apoptosis; Gap junction; Cytosolic calcium, Cancer tumors; Cancer therapy

\section{Introduction}

The purpose of this publication is to document and support the theory that the cancer cell is one without the ability to respond to homeostatic regulation. It is a disease of homeostatic dysfunction and the Intercellular Gap Junctions are the logical target for cancer chemoprevention and therapy ${ }^{[1]}$. Histological results and their interpretation of our calcium chelation experiments are presented. Emphasis is placed on the interruption of syncytial flow of ionic calcium $\left(\mathrm{Ca}^{++}\right)$ between unicellular muscle cells and its implications. Since Oxalic Acid (OA) had been found to be endogenous in humans ${ }^{[2]}$, we undertook a series of experiments to evaluate the effect, in vivo, of oxalates on the heart's intrinsic autonomic nervous system. OA was injected in the Anterior Right Ganglionated Plexus (ARGP) of the intrinsic cardiac autonomic nervous system in dogs ${ }^{[3]}$. Some of the oxalates infiltrated the underlying atrial myocardium. The histology of biopsy samples from those experiments was processed via a calcium specific von Kossa staining technique ${ }^{[4]}$, showing for the first time in vivo interruption of ionic calcium flow (syncytial flow) caused by chelation. 


\section{Methods}

Images of the von Kossa stained histological sections were viewed with a standard microscope and digitally downloaded into a file. No alterations were made to the original images. The staining kit and instructions were obtained from American Master Tech Scientific, Inc. Lodi, California, USA. In a previous experiment OA was selectively injected in the Right Atrial Ganglionated Plexus (RAGP) in concentrations of $100 \mathrm{mmol} / \mathrm{L}$. The total volume delivered was 0.5 milliliter $(\mathrm{mL})$ over one minute. The area covered was approximately $1 \mathrm{~cm}^{2}$.

\subsection{Von Kossa staining histology protocol}

Hematoxylin and eosin staining and von Kossa staining were carried out according to the procedure described by Sheehan DC and Hrapchak BB as referenced by The American Master Tech Von Kossa stain kit procedure. Hematoxylin/eosin and Von Kossa stains were performed on non-buffered formalin fixed paraffin-imbedded tissue sections, cut at $4 \mu$ thicknesses. The slides were deparaffinised using xylene, rinsed in distilled water and then placed in silver nitrate $5 \%$ solution for 40 minutes under a 100-watt incandescent lamp. Then the slides were rinsed in distilled water and placed in 5\% sodium thiosulfate solution for 2 to 3 minutes. Then they were rinsed in tap water and placed in nuclear fast red for 5 minutes. Finally, the slides were dehydrated in fresh absolute alcohol and then cleared in xylene and cover slipped. Additionally, control slides were placed in 1\% Hydrogen Chloride (HCL) solution for 5 minutes prior to the above Von Kossa staining procedure in order to confirm the presence of calcium. Calcium dissolves in acid urates or other salts do not. The calcium presence was confirmed as a series of individual or aggregated black spots.

Figure 1. In vivo experiment. No centrifugal force applied. Arrow denotes direction of physiological atrial myocardium cellular contraction force from right to left. (Arrow presumes direction of cellular contraction)

von Kossa staining or atrial myocardium after injection in vivo of $100 \mathrm{mM} / \mathrm{L}$ of Ammonium Oxalate. Magnification X40 A) Outline of intercalated discs B) Unstained atrial cytoplasm possibly at myocyte's bifurcation C) Granular chelated ionic calcium expressed as silver salt granules.

D) Cluster of individual chelated ionic calcium expressed also as silver precipitates granules of various sizes. E) Chelated intracellular calcium adhering coalescing against the Gap Junction. Example the gap junction selectivity towards $\mathrm{CaCO}_{3}$. Unpublished images from: Fan Y, Scherlag BJ, Embi A. et al. Neural effect of oxalic acid for atrial fibrillation therapy. (Abstract). Heart Rhythm. 2012; 9: S 258.

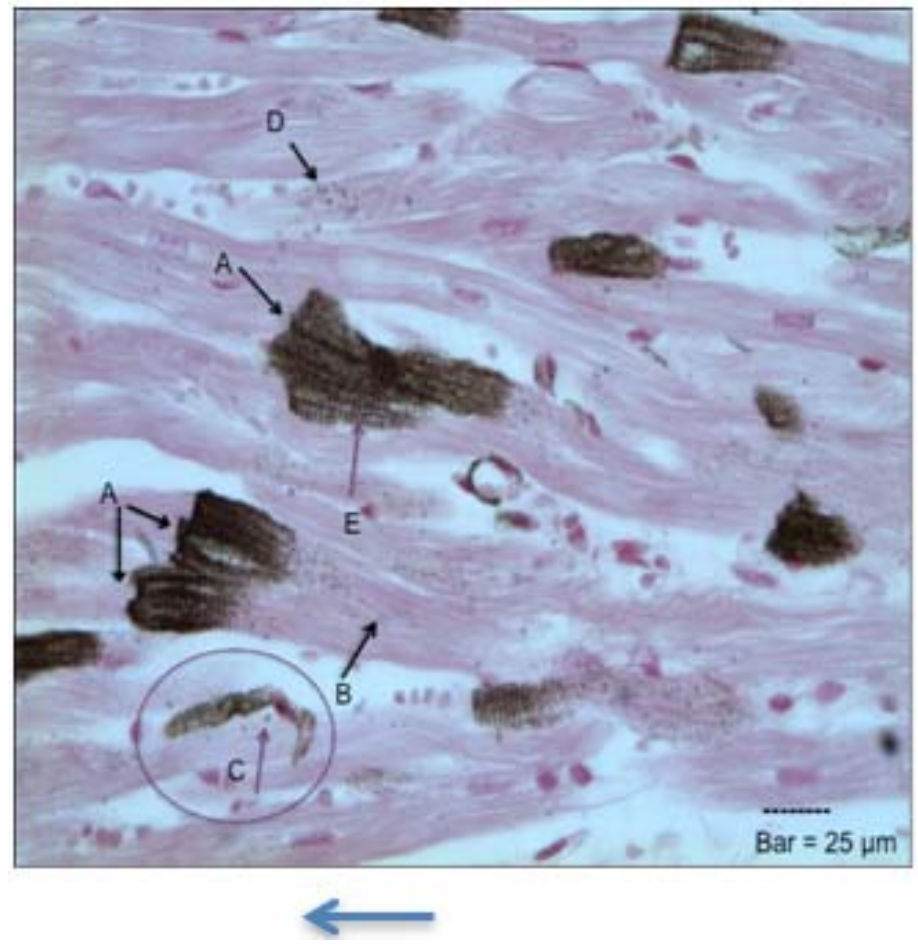

\subsection{Optical and photographic equipment}

For Figure 1, Leica DM2000 bright field transmission light microscope with 4 FLUOTAR lenses $(5 \times, 10 \times, 20 \times$ and $40 \times)$ and 2 N-Plan lenses $(2 \times$ and $60 \times$ high dry). 
Photographic Camera Moticam 1,000, 16 mm lens mounted on TL 160 BS adapter, digital images taken using corresponding Motic Images Plus software program. Images of the slides presented in this manuscript are originals, without any physical or digital alterations.

CONCENTRATION OF CALCIUM CHELATOR

OA: Molecular Weight $=126-\mathrm{pH} 1.6$

(100 mmol/L: $126 \mathrm{mg}$ in $10 \mathrm{~mL})$.

\section{Results and discussion}

The chelated intracellular atrial architecture was clearly outlined as shown in (E in Figure 1). The chelated atrial myocardium (stained black) was delineated by the chemical process of calcium chelation and subsequent staining. Calcium chelator, by the chemical process of ionic sequestration, converts the positive calcium ions $\left(\mathrm{Ca}^{++}\right)$into a calcium salt or calcium carbonate $\left(\mathrm{CaCO}_{3}\right)$ The von Kossa staining turns the $\mathrm{CaCO}_{3}$ black, resulting in the visualization of the intracellular calcium. The intercalated disc is clearly outlined (A in Figure 1) as well as other structures such as myofibrils are also detailed. The implications of these observations will be discussed further.

\subsection{Early research on oxalates and the cellular gap junction}

The early work done by Howell $\mathrm{WH}^{[5]}$ showed how oxalates could slow conduction in the neuro-muscular apparatus. He attributed the oxalate salts as the cause of the weakening. He most probably was witnessing the calcium chelation properties of oxalates. In 1961 Yokoyama ${ }^{[6]}$, injected the calcium chelator ehylenediaminetetraacetic acid (EDTA) in ventricular tissue, the purpose of his experiment was to physically dissociate the cardiac cells at the intercalated disc area. The anatomist Muir ${ }^{[7]}$, also dissociated the Purkinje cells, and using stainable glycogen in vitro in conjunction with centrifugal force claimed to have confirmed and expanded on Yokoyama's findings of the gap junction acting as a barrier

\subsection{Cell death and intracellular calcium levels}

It has been reported that increases in the cellular calcium levels is one of the factors triggering the initiation of cell death ${ }^{[8]}$. An increase of cytoplasmic $\mathrm{Ca}^{++}$of the heart tissue causes hypertrophy that resulted in heart dilatation or cardiomyopathy in transgenic mice ${ }^{[9]}$. Recently, the opposite, or a decrease of the available ionic calcium stores by calcium chelators (oxalates) was hypothesized to cause metastatic cancer cells death ${ }^{[10]}$. All three publications, Fan, Yokoyama and Embi show that the process of calcium chelation introduces a large molecular size $\left(\mathrm{CaCO}_{3}\right)$ within the cytoplasm. Muir demonstrated the same phenomenon by introducing in the cytoplasm the glycogen molecule. In all four aforementioned situations, the interruption of the normal syncytial ionic flow thru the gap junction was demonstrated.

\subsection{I ncidental in vivo chelated calcium coalescing at gap junction--the "log jam" effect}

The chelated samples obtained by Fan Y et al. by the OA in vivo injections were processed by our laboratory and showed similarities with the early-centrifuged images obtained by Muir and Yokoyama. Although our aim was to assess the neural effect of calcium chelation in the atrial ganglia, some of the injectate infiltrated the adjacent atrial tissue. We also show a "tailing effect" mimicking a "log jam" or coalescing of the larger molecules against the gap junction. This is observed on most of the chelated myocytes, as to reflect direction of flow or conduction (Figure 1). This finding is a demonstration of the gap junction property acting as a selective filter, and also a demonstration of the contractile direction of flow of the myocytes. It should be noted that unlike the in vitro anatomical approach of Muir (Figure 2), our in vivo approach presumes the physiological effect on atrial contractility. 
Figure 2. In vitro demonstration of gap junction selectivity towards the glycogen molecules. Purkinge Fibers after centrifugation. Notice the coalescing of the stained glycogen coalescing against the gap intercellular interalated discs. Gap Junction Selectivity towards molecular size is clearly demonstrated. (Arrow denotes centrifugal force) Image printed with permission from publisher: Muir AR. Further observations on the cellular structure of cardiac muscle $J$. Anat. Lond. 1965; 99: 27-46.

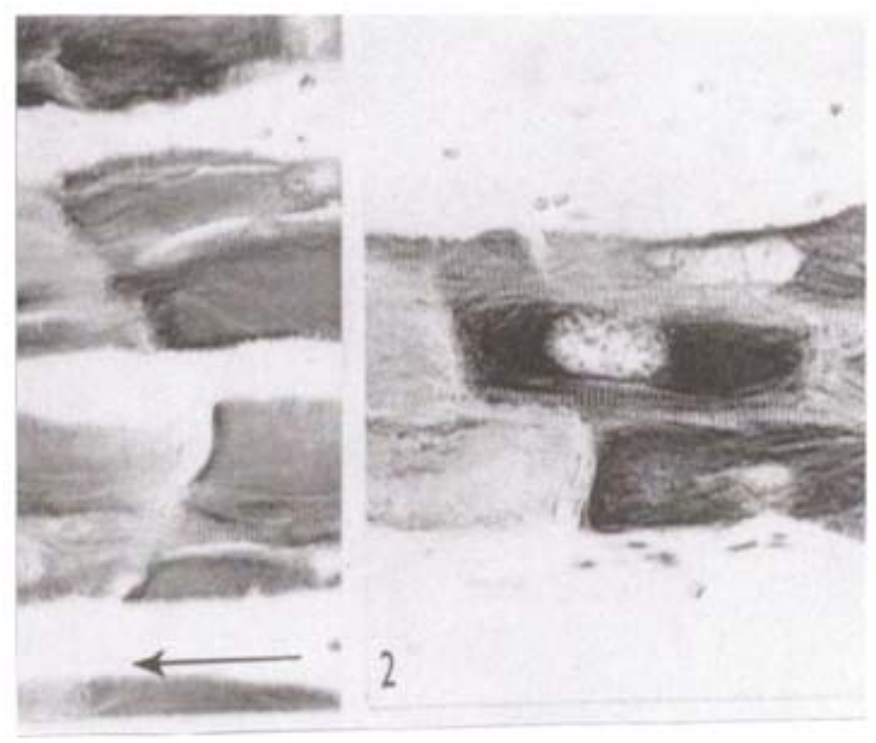

\subsection{Gap junction selectivity and molecular size}

Hervé JC ${ }^{[11]}$ comments, "Gap junctions allow cells to exchange ions and small molecules...Gap junction channels use "gating" mechanisms to open and close its pores in response to biological stimuli". De Mello WC ${ }^{[12]}$, referring to cardiac cell-to-cell communication stated "permeability of the nexus falls with increasing molecular weight of the compounds... The molecular size of the solvated molecule is, certainly, a more correct criterion for determining the permeability limit of the hydrophilic channels'. In our case the size of the molecules not allowed to flow by the gap junction confirms the previous statements in regards to size. The physical radius of the $\mathrm{CaCO}_{3}$ is much greater than the maximum reported pore opening of the gap junction. It has been reported that calcium in its ionic form measures a radius of 0.099 nanometers $(\mathrm{nm})$. On the other hand, the calcium carbonate molecules measure $\geqslant 10 \mathrm{~nm}^{[13]}$. Glycogen particles measure $24.9 \mathrm{~nm} \pm$ $1.8 \mathrm{~nm}^{[14]}$. The gap junction pores have a reported radius in the range of 0.6 to $1.5 \mathrm{~nm}^{[15]}$, thus not allowing passage of the larger calcium carbonate molecules and glycogen particles.

\subsection{Changes in intracellular calcium levels and cancer targeted calcium chelation of solid tumors}

Disruption of intracellular calcium levels has been found to trigger breast cancer cells apoptosis ${ }^{[16]}$. We show that calcium chelation also causes a disruption of the intracellular $\mathrm{Ca}^{++}$levels; and the delivery of calcium chelators via a targeted approach to existing brain metastatic melanoma tumors has been hypothesized to arrest the "protective" effect of Astrocytes towards malignant cells, thus increasing the efficiency of chemotherapeutic agents. Trosko in 1983 identified the gap junction as targets for cancer chemoprevention and therapy ${ }^{[17]}$. Trosko, recently in response to our targeted chelation paper stated (personal communication) that: " $\mathrm{Ca}^{++}$plays a major role in the regulation of gap junction function and, in some cases, it must play a role in its intercellular transfer through gap junctions controlling cell growth, differentiation and apoptosis, he continues " any test, especially in vivo, would have to be targeted"; furthermore he asks: Does internal $\mathrm{Ca}^{++}$chelation cause a release of sequestered $\mathrm{Ca}^{++}$from mitochondria or increased $\mathrm{Ca}^{++}$and influx in some way?

\subsection{Endoplasmic reticulum stress and calcium release}

Targeted chelation has been demonstrated by Fan et al. to cause a change in the essential extracellular ionic calcium in nerve tissue and intracellular in muscle tissue. One of the advantages of targeted solid tumor calcium chelation will be to 
possibly cause cell apoptosis of the malignant cells, while minimizing damage to the surrounding healthy tissue. As aforementioned, changes in intracellular cytosolic calcium levels trigger cell hypertrophy and death. On the other hand it has been proposed that cancer is a disease of homeostatic dysfunction; therefore a substantial change of available cytoplasmic $\mathrm{Ca}^{++}$by chelation increases the cytosolic cellular ionic calcium level. Does internal $\mathrm{Ca}^{++}$chelation cause a release of sequestered $\mathrm{Ca}^{++}$from mitochondria or increased $\mathrm{Ca}^{++}$influx? There is evidence supporting the aforementioned mechanism. It has been reported that in transgenic mouse, an increase in L-type calcium channels triggered a sustained ingress of cytosolic calcium. The focal calcium chelated images from that experiment (see Reference 9) shows striking similarities with the focal calcium chelated images obtained by Fan et al. (as shown in Figure 1).

\subsection{Clinical implications cancer as a disease of homeostatic dysfunction}

The interruption of the cytosolic intercellular communication by chelators can be defined as a down regulation of the gap junction intercellular communication (GJIC) of normal cells. Cancer cells lack growth control and cannot terminally differentiate if remained undisturbed. Intracellular calcium chelation could be the stimulus needed to disrupt this status $q u \bar{o}$ and initiate apotosis. Cancerous tumors as a disease of homeostatic dysfunction could be controlled by a targeted calcium chelation approach. As to the clinical prospects of this technique, a recent case study ${ }^{[18]}$ recently reported of inoperable retroperitoneal metastasized melanoma tumor could have benefited from a targeted delivery of oxalate chelators aimed at the tumor. This could be accomplished via different techniques, one of them is by intravascular delivery of superparamagnetic nanoparticles (MNP's) made of $\mathrm{Fe}_{3} \mathrm{O}_{4}$ (core), thermoresponsive polymeric hydrogel (shell) loaded with chelators. After delivery, these agents could be deployed ad libitum by an external magnetic source ${ }^{[19]}$.

\section{References}

[1] Trosko JE, Ruch RJ. Gap Junctions as Targets for Cancer Chemoprevention and Chemotherapy. Curr Drug Targets. 2002 Dec; 3(6): 465-82. PMid:12448698 http://dx.doi.org/10.2174/1389450023347371

[2] Lommel F. Uber die Herkunft der Oxalsaure im Harn. Dtsch. Arch. Kiln. Med. 1899; 599.

[3] Fan Y, BJ Scherlag, Embi A, Liu Y, Heng C, Mao J. Neural effect of oxalic acid for atrial fibrillation therapy. Heart Rhythm. 2012; 9: S 258.

[4] Sheehan DC, Hrapchak BB: Theory and Practice of Histotechnology. 2 nd edition- Columbus, OH- Battelle Memorial Institute. 1987.

[5] Howell WH. The action of oxalate solutions on nerve and Rigor Mortis. Journal of Physiology-Physiological Society. 1894; 16: 480.

[6] Yokoyama HA Jennings RB, Wartman WB. Intercalated discs of dog's myocardium. Exp Cell Res. 1961; $23: 29-44$. http://dx.doi.org/10.1016/0014-4827(61)90061-1

[7] Muir AR. Further observations on the cellular structure of cardiac muscle J. Anat. Lond. 1965; 99: 27-46.

[8] Pyrko P, Kardosh A, Liu Y-T, Soriano N, Xiong W, Chow RH. Calcium-activated endoplasmic reticulum stress as a major component of tumor cell death induced by 2, 5-dimethyl-celecoxib, a non-coxib analogue of celecoxib. Mol Cancer Ther. 2007; April 6; 1262. PMid:17431104 http://dx.doi.org/10.1158/1535-7163.MCT-06-0629

[9] Muth JN, Bodi I, Lewis W, Varadi G, Schwartz A. A Ca ${ }^{2+}$-Dependent Transgenic Model of Cardiac Hypertrophy. A Role for Protein Kinase C $\alpha$. Circulation. 2001; 103: 140-147. PMid:11136699 http://dx.doi.org/10.1161/01.CIR.103.1.140

[10] Embi A, Scherlag BJ, Embi PJ, Menes M, Po S. Targeted Cellular Ionic Calcium Chelation By Oxalates: Implications For The Treatment Of Tumor Cells. Cancer Cell International. 2012; 12: 51. PMid:23216811 http://dx.doi.org/10.1186/1475-2867-12-51

[11] HervéJC, Derangeon M.Gap-junction-mediated-cell-to-cell-communication. Cell and Tissue Research. 2012.

[12] De Mello WC. Intercellular communication in cardiac muscle. Circ Res. 1982; 51: 1-9. PMid:6282496 http://dx.doi.org/10.1161/01.RES.51.1.1

[13] Benzerara K, Menguy N, López-García P, Yoon T-H, Kazmierczak J, Tyliszczak T, Guyot F, et al. Nanoscale detection of organic signatures in carbonate microbialites. PNAS. 2006; 103 (25): 9440-9445. PMid:16772379 http://dx.doi.org/10.1073/pnas.0603255103

[14] Elsner P, Quistorff B, Hansen G, Grunnet N. Partly Ordered Synthesis and Degradation of Glycogen in Cultured Rat Myotubes. The Journal of Biological Chemistry. 2002; 277: 4831-4838. PMid:11724782 http://dx.doi.org/10.1074/jbc.M108226200 
[15] Müller DJ, Hand GM, Rangel A, Sosinsky GE. Conformational changes in surface structures of isolated connexin 26 gap junctions. EMBO J. 2002; 21(14): 3598-3607. PMid:12110573 http://dx.doi.org/10.1093/emboj/cdf365

[16] Baggott RR, Mohamed TM, Oceandy D, Holton M, Blanc MC, Roux-Soro SC, et al.. Disruption of the interaction between PMCA2 and calcineurin triggers apoptosis and enhances paclitaxel-induced cytotoxicity in breast cancer cells. Carcinogenesis. 2012 Dec; 33(12): 2362-8. PMid:22962307 http://dx.doi.org/10.1093/carcin/bgs282

[17] 2-Trosko JE, Chang CC and Medcalf A Mechanism of Tumor Promotion: Potential role of Intercellular Communication. Cancer Invest. 1983; 1: 511-526. PMid:6365277 http://dx.doi.org/10.3109/07357908309020276

[18] Arvind Randhawa, Niket Sonpal, Stephen Machnicki, Colette Spaccavento, Amory Novoselac A rare presentation of melanoma as a retroperitoneal mass: A case report a brief review of the literature. JST Journal of Solid Tumors. 2013; 3: 2.

[19] Yu L, Scherlag BJ, Dormer K, Nguyen KT, Pope C, Fung KM, Po SS. Autonomic denervation with magnetic nanoparticles. Circulation. 2010 Dec 21; 122(25): 2653-9. PMid:21135360 http://dx.doi.org/10.1161/CIRCULATIONAHA.110.940288 